\title{
Linearizability of Nonlinear Third-Order Ordinary Differential Equations by Using a Generalized Linearizing Transformation
}

\author{
E. Thailert and S. Suksern \\ Department of Mathematics, Faculty of Science, Naresuan University, Phitsanulok 65000, Thailand \\ Correspondence should be addressed to S. Suksern; s_suksern@hotmail.com
}

Received 5 May 2014; Accepted 5 August 2014; Published 14 August 2014

Academic Editor: Bin Zhou

Copyright (C) 2014 E. Thailert and S. Suksern. This is an open access article distributed under the Creative Commons Attribution License, which permits unrestricted use, distribution, and reproduction in any medium, provided the original work is properly cited.

We discuss the linearization problem of third-order ordinary differential equation under the generalized linearizing transformation. We identify the form of the linearizable equations and the conditions which allow the third-order ordinary differential equation to be transformed into the simplest linear equation. We also illustrate how to construct the generalized linearizing transformation. Some examples of linearizable equation are provided to demonstrate our procedure.

\section{Introduction}

There has been major interest in the nonlinear problems, since most equations are inherently nonlinear in nature. In general, the nonlinear problems are very difficult to solve explicitly. It is of interest to provide general criteria for the linearizability of nonlinear ordinary differential equations, as they can then be reduced to easily solvable equations. Therefore, the approach of investigating nonlinear ordinary differential equations via transforming to simpler ordinary differential equations becomes important and has been quite plentiful in analysis of physical problems. This includes the classical linearization problem of finding transformations that linearize a given ordinary differential equation. The linearization problem has been studied in many aspects. A short review can be found in $[1,2]$. The tools commonly used for solving the linearization problem are the transformations such as point transformation, contact transformation, reduction of order, differential substitution, and generalized Sundman transformation. For this paper, we employ the extension of the generalized Sundman transformations.

The linearization problem for a second-order ordinary differential equation was investigated with respect to a generalized Sundman transformation

$$
X=F(t, x), \quad d T=G(t, x) d t
$$

by Duarte et al. [3] earlier. They obtained the form of the linearizable equations and the conditions which allow the second-order ordinary differential equation to be transformed into the free particle equation. A characterization of these equations that can be linearized by means of generalized Sundman transformations in terms of first integral and procedure for construction of linearizing transformations has been given by Muriel and Romero [4]. In [5], Mustafa et al. gave a new characterization of linearizable equations in terms of the coefficients of ordinary differential equations and one auxiliary function. In [6], Nakpim and Meleshko pointed out that the solution of the linearization problem for a second-order ordinary differential equation via the generalized Sundman transformation considered earlier by Duarte et al. [3] using the Laguerre form is not complete.

The linearization problem for a third-order ordinary differential equation was also investigated with respect to a generalized Sundman transformation $[7,8]$. Criteria for a third-order ordinary differential equation to be equivalent to the linear equation $X^{\prime \prime \prime}(T)=0$ with respect to a Sundman transformation were presented in [8]. The generalized Sundman transformation was also applied for obtaining necessary and sufficient conditions for a third-order ordinary differential equation to be equivalent to a linear equation in the Laguerre form [6]. Some applications of the generalized Sundman transformation to ordinary differential equations 
were considered in [9] and earlier papers, summarized in the book [10].

The linearization problem of a fourth-order ordinary differential equation with respect to generalized Sundman transformations was studied in [11]. They found the necessary and sufficient conditions which allow the fourth-order ordinary differential equation to be transformed into the simplest linear equation.

In this work, we expose a more general transformation, that is, the extension of the generalized Sundman transformation

$$
X=F(t, x), \quad d T=G\left(t, x, x^{\prime}\right) d t
$$

This transformation was studied in [12-14] where they designated the transformation as the generalized linearizing transformation. They showed that this transformation can be utilized to linearize a wider class of nonlinear ordinary differential equations and, in particular, certain equations which cannot be linearized by the nonpoint and invertible point transformations. If the function $G$ in (2) is independent of the variable $x^{\prime}$, then it becomes a nonpoint transformation (vide (1)). On the other hand, if $G$ is a differentiable function, then it becomes an invertible point transformation. So (2) is a unified transformation as it includes nonpoint and invertible point transformations as special cases. An example of an equation which can be linearized by a transformation of the form (2) is given in [13]. It is worth noting that any second-order equation $x^{\prime \prime}=f\left(t, x, x^{\prime}\right)$ can be transformed by a transformation (2) into the free particle equation and that this is not so for third-order ordinary differential equations. Hence, the linearization problem using generalized linearizing transformations becomes interesting for ordinary differential equations of order greater than 2. In [12], the authors applied a particular class of transformations (2), where the function $G\left(t, x, x^{\prime}\right)$ is linear with respect to $x^{\prime}$.

We are now paying attention to the case where $G$ is a polynomial function in $x^{\prime}$ and in particular where it is linear in $x^{\prime}$ with coefficients which are arbitrary functions of $t$ and $x$. To be specific, we focus here on the case

$$
X=F(t, x), \quad d T=\left(G_{1}(t, x) x^{\prime}+G_{2}(t, x)\right) d t .
$$

Notice that for the case $G_{1}=0$, the generalized linearizing transformation becomes a generalized Sundman transformation, so that we assume $G_{1} \neq 0$.

The paper is organized as follows. In Section 2, the necessary conditions of linearization of a third-order ordinary differential equation are presented. In Section 3, we get the theorems that yield criteria for a third-order ordinary differential equation to be linearizable via generalized linearizing transformations. Examples which illustrate the procedure of using the linearization theorems are presented in Section 4.

\section{Necessary Conditions of Linearization}

Here we consider a nonlinear third-order ordinary differential equation

$$
x^{\prime \prime \prime}=f\left(t, x, x^{\prime}, x^{\prime \prime}\right) .
$$

Our goal in this section is to describe all equations (4) which are equivalent with respect to generalized linearizing transformations

$$
X=F(t, x), \quad d T=\left(G_{1}(t, x) x^{\prime}+G_{2}(t, x)\right) d t
$$

to a linear equation

$$
X^{\prime \prime \prime}(T)=0
$$

We begin with investigating the necessary conditions for linearization, that is, the general form of third-order equation (4) that can be obtained from a linear equation (6) by any generalized linearizing transformation (5).

Applying a generalized linearizing transformation (5), one obtains the following transformation of the third-order derivatives:

$$
X^{\prime}(T)=\frac{D_{t} F}{G_{1} x^{\prime}+G_{2}}=\frac{F_{t}+x^{\prime} F_{x}}{G_{1} x^{\prime}+G_{2}}=P\left(t, x, x^{\prime}\right),
$$

$$
\begin{aligned}
& =\frac{D_{t} P}{G_{1} x^{\prime}+G_{2}} \\
& =\frac{P_{t}+x^{\prime} P_{x}+x^{\prime \prime} P_{x^{\prime}}}{G_{1} x^{\prime}+G_{2}} \\
& =-\left(\left(G_{2 x} x^{\prime}+G_{1} x^{\prime \prime}+G_{2 t}+G_{1 x} x^{\prime 2}+G_{1 t} x^{\prime}\right) F_{t}\right. \\
& -\left(F_{t t}+F_{x x} x^{\prime 2}+2 F_{t x} x^{\prime}\right)\left(G_{1} x^{\prime}+G_{2}\right) \\
& \left.+\left(G_{2 x} x^{\prime 2}-G_{2} x^{\prime \prime}+G_{2 t} x^{\prime}+G_{1 x} x^{\prime 3}+G_{1 t} x^{\prime 2}\right) F_{x}\right) \\
& \times\left(G_{1} x^{\prime}+G_{2}\right)^{-3} \\
& =Q\left(t, x, x^{\prime}, x^{\prime \prime}\right) \text {, } \\
& X^{\prime \prime \prime}(T) \\
& =\left(\left(3 G_{2 x}^{2} x^{\prime 3}+G_{2}^{2} x^{\prime \prime \prime}+3 G_{2 t}^{2} x^{\prime}+3 G_{1 x}^{2} x^{15}\right.\right. \\
& +3 G_{1 t}^{2} x^{\prime 3}+\left(x^{\prime} x^{\prime \prime \prime}-3 x^{\prime \prime 2}\right) G_{1} G_{2} \\
& +2\left(G_{1} x^{\prime}-2 G_{2}\right) G_{2 x} x^{\prime} x^{\prime \prime} \\
& -\left(G_{2 t t}+G_{2 x x} x^{\prime 2}+2 G_{2 t x} x^{\prime}+G_{1 x x} x^{\prime 3}\right. \\
& \left.+G_{1 t t} x^{\prime}+2 G_{1 t x} x^{\prime 2}\right)\left(G_{1} x^{\prime}+G_{2}\right) x^{\prime} \\
& +3\left(\left(G_{1} x^{\prime}-G_{2}\right) x^{\prime \prime}+2 G_{2 x} x^{\prime 2}\right) G_{2 t} \\
& +6\left(G_{2 x} x^{\prime 2}-G_{2} x^{\prime \prime}+G_{2 t} x^{\prime}\right) G_{1 x} x^{\prime 2}
\end{aligned}
$$




$$
\begin{aligned}
& +\left(\left(G_{1} x^{\prime}-5 G_{2}\right) x^{\prime \prime}+6 G_{2 x} x^{\prime 2}+6 G_{2 t} x^{\prime}\right. \\
& \left.\left.+6 G_{1 x} x^{\prime 3}\right) G_{1 t} x^{\prime}\right) F_{x} \\
& -\left(3\left(G_{2 t}+G_{2 x} x^{\prime}+G_{1 x} x^{\prime 2}+G_{1 t} x^{\prime}+G_{1} x^{\prime \prime}\right) F_{t t}\right. \\
& -\left(\left(3 F_{t t x}+F_{x x x} x^{\prime 2}\right) x^{\prime}+F_{t t t}+3 F_{t x x} x^{\prime 2}\right) \\
& \times\left(G_{1} x^{\prime}+G_{2}\right) \\
& +3\left(\left(G_{2 t}+G_{2 x} x^{\prime}+G_{1 x} x^{\prime 2}+G_{1 t} x^{\prime}\right) x^{\prime}-G_{2} x^{\prime \prime}\right) \\
& \times F_{x x} x^{\prime}+3\left(2\left(G_{2 t}+G_{2 x} x^{\prime}+G_{1 x} x^{\prime 2}+G_{1 t} x^{\prime}\right) x^{\prime}\right. \\
& \left.\left.+\left(G_{1} x^{\prime}-G_{2}\right) x^{\prime \prime}\right) F_{t x}\right)\left(G_{1} x^{\prime}+G_{2}\right) \\
& -\left(\left(G_{2 t t}+G_{2 x x} x^{\prime 2}+2 G_{2 t x} x^{\prime}+G_{1 x x} x^{\prime 3}\right.\right. \\
& \left.+G_{1 t t} x^{\prime}+2 G_{1 t x} x^{\prime 2}\right) \\
& \times\left(G_{1} x^{\prime}+G_{2}\right) \\
& -\left(3 G_{2 x}^{2} x^{\prime 2}-G_{1} G_{2} x^{\prime \prime \prime}+3 G_{2 t}^{2}+3 G_{1 x}^{2} x^{\prime 4}\right. \\
& \left.+3 G_{1 t}^{2} x^{\prime 2}-\left(x^{\prime} x^{\prime \prime \prime}-3 x^{\prime \prime 2}\right) G_{1}^{2}\right) \\
& \text { - }\left(5 G_{1} x^{\prime}-G_{2}\right) G_{2 x} x^{\prime \prime} \\
& -6\left(G_{2 x} x^{\prime}+G_{1} x^{\prime \prime}\right) G_{2 t} \\
& -3\left(\left(G_{1} x^{\prime}-G_{2}\right) x^{\prime \prime}+2 G_{2 x} x^{\prime 2}+2 G_{2 t} x^{\prime}\right) \\
& \times G_{1 x} x^{\prime}-2\left(\left(2 G_{1} x^{\prime}-G_{2}\right) x^{\prime \prime}+3 G_{2 x} x^{\prime 2}\right. \\
& \left.\left.\left.+3 G_{2 t} x^{\prime}+3 G_{1 x} x^{\prime 3}\right) G_{1 t}\right) F_{t}\right) \\
& \times\left(G_{1} x^{\prime}+G_{2}\right)^{-5} \\
& =R\left(t, x, x^{\prime}, x^{\prime \prime}, x^{\prime \prime \prime}\right) \text {, }
\end{aligned}
$$

where $D_{t}=\partial / \partial t+x^{\prime}(\partial / \partial x)+x^{\prime \prime}\left(\partial / \partial x^{\prime}\right)+x^{\prime \prime \prime}\left(\partial / \partial x^{\prime \prime}\right)+\cdots$ is a total of derivatives. Substituting the resulting expression in linear equation (6) and setting $r=G_{2} / G_{1}, K=F_{t}-F_{x} r$, we arrive at the following equation:

$$
\begin{aligned}
x^{\prime \prime \prime}+ & \frac{1}{x^{\prime}+r} \\
& \times\left[-3 x^{\prime \prime 2}+\left(A_{2} x^{\prime 2}+A_{1} x^{\prime}+A_{0}\right) x^{\prime \prime}\right. \\
& \left.\quad+B_{5} x^{\prime 5}+B_{4} x^{\prime 4}+B_{3} x^{\prime 3}+B_{2} x^{\prime 2}+B_{1} x^{\prime}+B_{0}\right] \\
& =
\end{aligned}
$$

where $A_{i}(i=0,1,2)$ and $B_{j}(j=0,1, \ldots, 5)$ are functions of $t$ and $x$ determined as follows:

$$
\begin{aligned}
& A_{2}=\left(3\left(\left(F_{t x}-F_{x x} r\right) G_{1}-F_{t} G_{1 x}\right)\right. \\
& \left.+\left(2\left(2 G_{1 x} r-r_{x} G_{1}\right)-G_{1 t}\right) F_{x}\right) /\left(K G_{1}\right), \\
& A_{1}=-\left(\left(2 G_{1 x} r+5 r_{x} G_{1}+4 G_{1 t}\right) F_{t}\right. \\
& -3\left(F_{t t}-F_{x x} r^{2}\right) G_{1} \\
& +\left(\left(3 r_{t}-4 r_{x} r\right) G_{1}\right. \\
& \left.\left.-4 G_{1 x} r^{2}-2 G_{1 t} r\right) F_{x}\right) /\left(K G_{1}\right), \\
& A_{0}=-\left(3 F_{t x} G_{1} r^{2}-3 F_{t t} G_{1} r+4 F_{t} G_{1 t} r\right. \\
& -F_{t} G_{1 x} r^{2}+6 F_{t} r_{t} G_{1}-F_{t} r_{x} G_{1} r \\
& \left.-3 F_{x} G_{1 t} r^{2}-3 F_{x} r_{t} G_{1} r\right) /\left(K G_{1}\right), \\
& B_{5}=-\frac{\left(\left(F_{x x x} G_{1}-3 F_{x x} G_{1 x}\right) G_{1}-\left(G_{1 x x} G_{1}-3 G_{1 x}^{2}\right) F_{x}\right)}{\left(K G_{1}^{2}\right)}, \\
& B_{4}=\left(3\left(G_{1 t}+2 G_{1 x} r+r_{x} G_{1}\right) F_{x x} G_{1}\right. \\
& +\left(G_{1 x x} G_{1}-3 G_{1 x}^{2}\right) F_{t} \\
& +\left(2 G_{1 t x} G_{1}-6 G_{1 t} G_{1 x}+2 G_{1 x x} G_{1} r-6 G_{1 x}^{2} r\right. \\
& \left.-4 G_{1 x} r_{x} G_{1}+r_{x x} G_{1}^{2}\right) F_{x} \\
& \left.+\left(2\left(3 F_{t x} G_{1 x}-F_{x x x} G_{1} r\right)-3 F_{t x x} G_{1}\right) G_{1}\right) \\
& \times\left(K G_{1}^{2}\right)^{-1}, \\
& B_{3}=\left(\left(3 F_{t t} G_{1 x}-F_{x x x} G_{1} r^{2}-3 F_{t t x} G_{1}-6 F_{t x x} G_{1} r\right.\right. \\
& \left.+6\left(G_{1 t}+2 G_{1 x} r+r_{x} G_{1}\right) F_{t x}\right) G_{1} \\
& +\left(2 G_{1 t x} G_{1}-6 G_{1 t} G_{1 x}+2 G_{1 x x} G_{1} r-6 G_{1 x}^{2} r\right. \\
& \left.-4 G_{1 x} r_{x} G_{1}+r_{x x} G_{1}^{2}\right) F_{t} \\
& +3\left(\left(G_{1 x} r+r_{x} G_{1}+G_{1 t}\right) r+G_{1 t} r+r_{t} G_{1}\right) F_{x x} G_{1} \\
& +\left(\left(2 G_{1 t x} r+G_{1 t t}\right) G_{1}-3\left(G_{1 x} r+r_{x} G_{1}\right)^{2}\right. \\
& -6\left(G_{1 t} r+r_{t} G_{1}\right) G_{1 x} \\
& -3\left(2\left(G_{1 x} r+r_{x} G_{1}\right)+G_{1 t}\right) G_{1 t} \\
& +\left(2 G_{1 x} r_{x}+r_{x x} G_{1}+G_{1 x x} r\right) G_{1} r
\end{aligned}
$$




$$
\begin{aligned}
& \left.\left.+2\left(G_{1 x} r_{t}+r_{t x} G_{1}+G_{1 t} r_{x}+G_{1 t x} r\right) G_{1}\right) F_{x}\right) \\
& \times\left(K G_{1}^{2}\right)^{-1} \\
& B_{2}=\left(\left(\left(2 G_{1 t x} r+G_{1 t t}\right) G_{1}-3\left(G_{1 x} r+r_{x} G_{1}\right)^{2}\right.\right. \\
& -6\left(G_{1 t} r+r_{t} G_{1}\right) G_{1 x} \\
& -3\left(2\left(G_{1 x} r+r_{x} G_{1}\right)+G_{1 t}\right) G_{1 t} \\
& +\left(2 G_{1 x} r_{x}+r_{x x} G_{1}+G_{1 x x} r\right) G_{1} r \\
& \left.+2\left(G_{1 x} r_{t}+r_{t x} G_{1}+G_{1 t} r_{x}+G_{1 t x} r\right) G_{1}\right) F_{t} \\
& -\left(\left(6\left(G_{1 x} r+r_{x} G_{1}+G_{1 t}\right)\right.\right. \\
& \times\left(G_{1 t} r+r_{t} G_{1}\right)-G_{1 t t} G_{1} r \\
& -\left(2 G_{1 t} r_{t}+r_{t t} G_{1}+G_{1 t t} r\right) G_{1} \\
& \left.-2\left(G_{1 x} r_{t}+r_{t x} G_{1}+G_{1 t} r_{x}+G_{1 t x} r\right) G_{1} r\right) F_{x} \\
& +\left(\left(3 F_{t x x} r^{2}+F_{t t t}\right) G_{1}\right. \\
& -3\left(G_{1 t}+2 G_{1 x} r+r_{x} G_{1}\right) F_{t t} \\
& -3\left(\left(G_{1 t} r+r_{t} G_{1}\right) F_{x x}-2 F_{t t x} G_{1}\right) r \\
& -6\left(\left(G_{1 x} r+r_{x} G_{1}+G_{1 t}\right) r+G_{1 t} r+r_{t} G_{1}\right) \\
& \left.\left.\left.\times F_{t x}\right) G_{1}\right)\right) \\
& \times\left(K G_{1}^{2}\right)^{-1} \\
& B_{1}=-\left(\left(6\left(G_{1 x} r+r_{x} G_{1}+G_{1 t}\right)\left(G_{1 t} r+r_{t} G_{1}\right)\right.\right. \\
& -G_{1 t t} G_{1} r-\left(2 G_{1 t} r_{t}+r_{t t} G_{1}+G_{1 t t} r\right) G_{1} \\
& \left.-2\left(G_{1 x} r_{t}+r_{t x} G_{1}+G_{1 t} r_{x}+G_{1 t x} r\right) G_{1} r\right) F_{t} \\
& -\left(\left(3\left(\left(G_{1 x} r+r_{x} G_{1}+G_{1 t}\right) r+G_{1 t} r+r_{t} G_{1}\right) F_{t t}\right.\right. \\
& -\left(\left(2 F_{t t t}+3 F_{t t x} r\right) G_{1}\right. \\
& \left.\left.-6\left(G_{1 t} r+r_{t} G_{1}\right) F_{t x}\right) r\right) G_{1} \\
& +\left(\left(2 G_{1 t} r_{t}+r_{t t} G_{1}+G_{1 t t} r\right) G_{1} r\right. \\
& \left.\left.\left.-3\left(G_{1 t} r+r_{t} G_{1}\right)^{2}\right) F_{x}\right)\right)
\end{aligned}
$$

$$
\begin{aligned}
B_{0}=( & \left(\left(2 G_{1 t} r_{t}+r_{t t} G_{1}+G_{1 t t} r\right) G_{1} r\right. \\
& \left.-3\left(G_{1 t} r+r_{t} G_{1}\right)^{2}\right) F_{t} \\
+ & \left.\left(3\left(G_{1 t} r+r_{t} G_{1}\right) F_{t t}-F_{t t t} G_{1} r\right) G_{1} r\right) /\left(K G_{1}^{2}\right) .
\end{aligned}
$$

Thus, we proved the theorem.

Theorem 1. Any third-order ordinary differential equation (4) obtained from a linear equation (6) by a generalized linearizing transformation (5) has to be in the form (8).

\section{Formulation of the Linearization Theorem}

We have shown in the previous section that every linearizable third-order ordinary differential equation belongs to the class of equations (8). In this section, we formulate the main theorems containing necessary and sufficient conditions for linearization as well as the methods for constructing the linearizing transformations.

For obtaining sufficient conditions, one has to solve the compatibility problem. Consider the representations of the coefficients $A_{i}$ and $B_{i}$ through the unknown functions $F$ and $G_{1}$. According to our notation $K=F_{t}-F_{x} r$, we define the derivative $F_{t}$ as

$$
F_{t}=F_{x} r+K
$$

From (9), one can find the derivatives

$$
\begin{gathered}
K_{x}=\frac{\left(F_{x} G_{1 t}-F_{x} G_{1 x} r-F_{x} r_{x} G_{1}+3 G_{1 x} K+A_{2} G_{1} K\right)}{\left(3 G_{1}\right)}, \\
K_{t}=\left(F_{x} G_{1 t} r-F_{x} G_{1 x} r^{2}-F_{x} r_{x} G_{1} r+4 G_{1 t} K-G_{1 x} K r\right. \\
\left.+G_{1} K\left(5 r_{x}+A_{1}-A_{2} r\right)\right) /\left(3 G_{1}\right) .
\end{gathered}
$$

From (10), one obtains the condition

$$
r_{t}=\frac{\left(6 r_{x} r-A_{0}+A_{1} r-A_{2} r^{2}\right)}{6} .
$$

Equation (11) defines the derivative

$$
F_{x x x}=\frac{\left(3 F_{x x} G_{1 x} G_{1}+F_{x} G_{1 x x} G_{1}-3 F_{x} G_{1 x}^{2}-B_{5} G_{1}^{2} K\right)}{G_{1}^{2}} .
$$

So that equation (12) becomes

$$
\begin{aligned}
& 6 F_{x x} G_{1 t} G_{1}-6 F_{x x} G_{1 x} G_{1} r-6 F_{x x} r_{x} G_{1}^{2} \\
& \quad+3 F_{x} G_{1 t x} G_{1}-12 F_{x} G_{1 t} G_{1 x} \\
& \quad-F_{x} G_{1 t} A_{2} G_{1}-3 F_{x} G_{1 x x} G_{1} r+12 F_{x} G_{1 x}^{2} r
\end{aligned}
$$




$$
\begin{aligned}
& +F_{x} G_{1 x} G_{1}\left(6 r_{x}+A_{2} r\right) \\
& +F_{x} G_{1}^{2}\left(-3 r_{x x}+r_{x} A_{2}\right)-6 G_{1 x x} G_{1} K+9 G_{1 x}^{2} K \\
& +G_{1}^{2} K\left(-3 A_{2 x}-A_{2}^{2}-3 B_{4}+15 B_{5} r\right)=0 .
\end{aligned}
$$

The compatibility analysis depends on the value of $F_{x}$. A complete study of all cases is given here.

3.1. Case $F_{x}=0$. In this case, the forms of derivatives $F_{t}, K_{x}$, and $K_{t}$ become

$$
\begin{gathered}
F_{t}=K, \\
K_{x}=\frac{\left(3 G_{1 x}+A_{2} G_{1}\right) K}{\left(3 G_{1}\right)}, \\
K_{t}=\frac{\left(4 G_{1 t}-G_{1 x} r+G_{1}\left(5 r_{x}+A_{1}-A_{2} r\right)\right) K}{\left(3 G_{1}\right)} .
\end{gathered}
$$

Substituting $F_{x}$ into $F_{x x x}$, one arrives at the condition

$$
B_{5}=0 \text {. }
$$

Comparing the mixed derivatives $\left(F_{x}\right)_{t}=\left(F_{t}\right)_{x}$, one gets the derivative

$$
G_{1 x}=\frac{-\left(A_{2} G_{1}\right)}{3} .
$$

In this case, $\left(F_{x x x}\right)_{t}=\left(F_{t}\right)_{x x x}$ is satisfied. Equations (12) and (13) give the conditions

$$
\begin{gathered}
A_{2 x}=\frac{\left(-2 A_{2}^{2}-9 B_{4}\right)}{3}, \\
r_{x x}=\frac{\left(-9 A_{1 x}+6 A_{2 t}+3 r_{x} A_{2}-3 A_{1} A_{2}-2 A_{2}^{2} r-9 B_{3}\right)}{36} .
\end{gathered}
$$

Comparing the mixed derivative $\left(K_{t}\right)_{x}=\left(K_{x}\right)_{t}$, one obtains the condition

$$
A_{1 x}=\frac{\left(-6 A_{2 t}-3 r_{x} A_{2}-5 A_{1} A_{2}+2 A_{2}^{2} r-15 B_{3}+24 B_{4} r\right)}{3} .
$$

Equation (14) provides the derivative

$$
G_{1 t t}=\frac{\left(2250 G_{1 t}^{2}+150 G_{1 t} G_{1} h_{1}+G_{1}^{2} h_{2}\right)}{\left(1350 G_{1}\right)},
$$

where

$$
\begin{gathered}
h_{1}=15 r_{x}+3 A_{1}-2 A_{2} r, \\
h_{2}=-225 A_{0 x}-1350 A_{1 t}-1350 A_{2 t} r-1050 A_{0} A_{2} \\
-477 A_{1}^{2}+516 A_{1} A_{2} r+33 A_{1} h_{1}-432 A_{2}^{2} r^{2} \\
-57 A_{2} h_{1} r-4050 B_{2}+4275 B_{3} r-4275 B_{4} r^{2}-8 h_{1}^{2} .
\end{gathered}
$$

The relation $\left(r_{x}\right)_{x}=r_{x x}$ gives the condition

$$
h_{1 x}=4 A_{2 t} .
$$

Comparing the mixed derivative $\left(G_{1 t t}\right)_{x}=\left(G_{1 x}\right)_{t t}$, one arrives at the condition

$$
A_{2 t t}=\frac{\left(50 A_{2 t} h_{1}-h_{2 x}\right)}{450} .
$$

Solving (15), one finds the conditions

$$
\begin{aligned}
& A_{0 t} \\
& \begin{aligned}
=( & 15930 A_{1 t} r+15930 A_{2 t} r^{2}-1260 h_{1 x} r \\
& -1575 A_{0} A_{1}+11970 A_{0} A_{2} r+5517 A_{1}^{2} r \\
& -5697 A_{1} A_{2} r^{2}-558 A_{1} h_{1} r+4986 A_{2}^{2} r^{3} \\
& +504 A_{2} h_{1} r^{2}-8100 B_{1}+48600 B_{2} r \\
& \left.-48600 B_{3} r^{2}+48600 B_{4} r^{3}+148 h_{1}^{2} r+8 h_{2} r\right) / 1350
\end{aligned}
\end{aligned}
$$

$$
\begin{aligned}
& B_{0} \\
& =\left(-3240 A_{1 t} r^{2}-3240 A_{2 t} r^{3}+180 h_{1 x} r^{2}\right. \\
& \quad-135 A_{0}^{2}+270 A_{0} A_{1} r-2430 A_{0} A_{2} r^{2} \\
& \quad-1107 A_{1}^{2} r^{2}+1134 A_{1} A_{2} r^{3}+108 A_{1} h_{1} r^{2} \\
& \quad-999 A_{2}^{2} r^{4}-108 A_{2} h_{1} r^{3}+1620 B_{1} r-9720 B_{2} r^{2} \\
& \left.+9720 B_{3} r^{3}-9720 B_{4} r^{4}-28 h_{1}^{2} r^{2}-2 h_{2} r^{2}\right) / 1620 .
\end{aligned}
$$

3.2. Case $F_{x} \neq 0$. From (20) and (13), one obtains the derivatives

$$
\begin{aligned}
G_{1 t x}=( & -6 F_{x x} G_{1 t} G_{1}+6 F_{x x} G_{1 x} G_{1} r+6 F_{x x} r_{x} G_{1}^{2} \\
& +12 F_{x} G_{1 t} G_{1 x}+F_{x} G_{1 t} A_{2} G_{1}+3 F_{x} G_{1 x x} G_{1} r \\
& -12 F_{x} G_{1 x}^{2} r+F_{x} G_{1 x} G_{1}\left(-6 r_{x}-A_{2} r\right) \\
& +F_{x} G_{1}^{2}\left(3 r_{x x}-r_{x} A_{2}\right)+6 G_{1 x x} G_{1} K \\
& \left.-9 G_{1 x}^{2} K+G_{1}^{2} K\left(3 A_{2 x}+A_{2}^{2}+3 B_{4}-15 B_{5} r\right)\right) \\
\times & \left(3 F_{x} G_{1}\right)^{-1}, \\
G_{1 t t}=( & -24 F_{x x} F_{x} G_{1 t} G_{1} r+24 F_{x x} F_{x} G_{1 x} G_{1} r^{2} \\
& +24 F_{x x} F_{x} r_{x} G_{1}^{2} r-24 F_{x x} G_{1 t} G_{1} K+24 F_{x x} G_{1 x} G_{1} K r \\
& +24 F_{x x} r_{x} G_{1}^{2} K+14 F_{x}^{2} G_{1 t}^{2}+20 F_{x}^{2} G_{1 t} G_{1 x} r
\end{aligned}
$$




$$
\begin{aligned}
& +2 F_{x}^{2} G_{1 t} G_{1}\left(r_{x}+A_{1}\right)+6 F_{x}^{2} G_{1 x x} G_{1} r^{2}-34 F_{x}^{2} G_{1 x}^{2} r^{2} \\
& +F_{x}^{2} G_{1 x} G_{1}\left(-26 r_{x} r-A_{0}-A_{1} r-A_{2} r^{2}\right) \\
& +F_{x}^{2} G_{1}^{2}\left(-A_{0 x}+A_{1 x} r-A_{2 x} r^{2}+12 r_{x x} r\right. \\
& \left.\quad-4 r_{x}^{2}-r_{x} A_{1}-2 r_{x} A_{2} r\right) \\
& +24 F_{x} G_{1 t} G_{1 x} K+24 F_{x} G_{1 x x} G_{1} K r-60 F_{x} G_{1 x}^{2} K r \\
& -24 F_{x} G_{1 x} r_{x} G_{1} K \\
& +2 F_{x} G_{1}^{2} K\left(3 A_{1 x}+18 r_{x x}-3 r_{x} A_{2}\right. \\
& \left.\quad+A_{1} A_{2}+3 B_{3}-6 B_{4} r\right)+24 G_{1 x x} G_{1} K^{2} \\
& \left.+36 G_{1 x}^{2} K^{2}+4 G_{1}^{2} K^{2}\left(3 A_{2 x}+A_{2}^{2}+3 B_{4}-15 B_{5} r\right)\right) \\
& \times\left(6 F_{x}^{2} G_{1}\right)^{-1} \cdot
\end{aligned}
$$

Comparing the mixed derivative $\left(K_{t}\right)_{x}=\left(K_{x}\right)_{t}$, one obtains

$$
\begin{aligned}
G_{1 x x}= & 6 F_{x x} G_{1 t} G_{1} K-6 F_{x x} G_{1 x} G_{1} K r \\
& -6 F_{x x} r_{x} G_{1}^{2} K-F_{x}^{2} G_{1 t}^{2}+2 F_{x}^{2} G_{1 t} G_{1 x} r \\
& +2 F_{x}^{2} G_{1 t} r_{x} G_{1}-F_{x}^{2} G_{1 x}^{2} r^{2}-2 F_{x}^{2} G_{1 x} r_{x} G_{1} r \\
& -F_{x}^{2} r_{x}^{2} G_{1}^{2}-6 F_{x} G_{1 t} G_{1 x} K+6 F_{x} G_{1 x}^{2} K r \\
& +6 F_{x} G_{1 x} r_{x} G_{1} K \\
& +F_{x} G_{1}^{2} K\left(-3 A_{2 t}+3 A_{2 x} r-A_{1} A_{2}+2 A_{2}^{2} r\right. \\
& \left.\quad-3 B_{3}+12 B_{4} r-30 B_{5} r^{2}\right) \\
& \left.+9 G_{1 x}^{2} K^{2}+G_{1}^{2} K^{2}\left(-3 A_{2 x}-A_{2}^{2}-3 B_{4}+15 B_{5} r\right)\right) \\
\times & \left(6 G_{1} K^{2}\right)^{-1} .
\end{aligned}
$$

Equation (14) becomes

$$
F_{x} s_{1}+2 K s_{2}=0
$$

where

$$
\begin{aligned}
s_{1}= & -6 A_{1 t}+6 A_{1 x} r+12 A_{2 t} r-12 A_{2 x} r^{2} \\
& -5 A_{0} A_{2}-2 A_{1}^{2}+13 A_{1} A_{2} r-13 A_{2}^{2} r^{2} \\
& -18 B_{2}+54 B_{3} r-108 B_{4} r^{2}+180 B_{5} r^{3}, \\
s_{2}= & -3 A_{1 x}+6 A_{2 t}-18 r_{x x}+3 r_{x} A_{2} \\
& +A_{1} A_{2}-2 A_{2}^{2} r+3 B_{3}-12 B_{4} r+30 B_{5} r^{2} .
\end{aligned}
$$

Further analysis of the compatibility depends on value of $s_{1}$ in (35): it is separated into two cases; that is, $s_{1}=0$ and $s_{1} \neq 0$.
3.2.1. Case $s_{1} \neq 0$. From (35), one finds

$$
F_{x}=-\frac{\left(2 K s_{2}\right)}{s_{1}}
$$

Since this case $F_{x} \neq 0$, then $s_{2} \neq 0$ too. Comparing the mixed derivatives $\left(F_{x}\right)_{t}=\left(F_{t}\right)_{x}$, one gets the derivative

$$
G_{1 t}=\frac{\left(3 G_{1 x} s_{1}\left(2 r s_{2}-s_{1}\right)+G_{1} s_{3}\right)}{\left(6 s_{1} s_{2}\right)}
$$

where

$$
\begin{aligned}
s_{3}= & -6 r_{x} s_{1} s_{2}+6 s_{1 t} s_{2}-6 s_{1 x} r s_{2}-6 s_{2 t} s_{1} \\
& +6 s_{2 x} r s_{1}-2 A_{1} s_{1} s_{2}+4 A_{2} r s_{1} s_{2}-A_{2} s_{1}^{2} .
\end{aligned}
$$

Substituting $F_{x}$ into $F_{x x x}, G_{1 t}$ into $G_{1 t x}$ and $G_{1 t t}$, one arrives at the conditions

$$
\begin{aligned}
s_{2 x x}=( & -12 A_{2 t} s_{1}^{2} s_{2}^{2}+12 A_{2 x} r s_{1}^{2} s_{2}^{2}-6 A_{2 x} s_{1}^{3} s_{2} \\
& +36 r_{x} s_{1 x} s_{1} s_{2}^{2}-36 r_{x} s_{2 x} s_{1}^{2} s_{2}-12 r_{x} A_{2} s_{1}^{2} s_{2}^{2} \\
& +18 s_{1 x x} s_{1}^{2} s_{2}-36 s_{1 x}^{2} s_{1} s_{2}+36 s_{1 x} s_{2 x} s_{1}^{2} \\
& +12 s_{1 x} A_{2} s_{1}^{2} s_{2}-6 s_{1 x} s_{2} s_{3}-12 s_{2 x} A_{2} s_{1}^{3} \\
& +6 s_{2 x} s_{1} s_{3}-4 A_{1} A_{2} s_{1}^{2} s_{2}^{2}+8 A_{2}^{2} r s_{1}^{2} s_{2}^{2} \\
& -2 A_{2}^{2} s_{1}^{3} s_{2}+2 A_{2} s_{1} s_{2} s_{3}-12 B_{3} s_{1}^{2} s_{2}^{2} \\
& \left.+48 B_{4} r s_{1}^{2} s_{2}^{2}-120 B_{5} r^{2} s_{1}^{2} s_{2}^{2}+9 B_{5} s_{1}^{4}\right) \\
\times & \left(18 s_{1}^{3}\right)^{-1},
\end{aligned}
$$

$$
s_{3 x}=\left(-6 A_{1 x} s_{1}^{3} s_{2}-6 A_{2 t} s_{1}^{3} s_{2}+18 A_{2 x} r s_{1}^{3} s_{2}\right.
$$$$
-9 A_{2 x} s_{1}^{4}+36 r_{x}^{2} s_{1}^{2} s_{2}^{2}-36 r_{x} s_{1 x} s_{1}^{2} s_{2}+36 r_{x} s_{2 x} s_{1}^{3}
$$$$
+6 r_{x} A_{2} s_{1}^{3} s_{2}-12 r_{x} s_{1} s_{2} s_{3}+12 s_{1 x} s_{1} s_{3}
$$$$
-4 A_{1} A_{2} s_{1}^{3} s_{2}+8 A_{2}^{2} r s_{1}^{3} s_{2}-3 A_{2}^{2} s_{1}^{4}-12 B_{3} s_{1}^{3} s_{2}
$$$$
+48 B_{4} r s_{1}^{3} s_{2}-9 B_{4} s_{1}^{4}-120 B_{5} r^{2} s_{1}^{3} s_{2}
$$$$
\left.+45 B_{5} r s_{1}^{4}-2 s_{1}^{3} s_{2}^{2}+s_{3}^{2}\right) /\left(6 s_{1}^{2}\right),
$$

$$
\begin{aligned}
s_{3 t}=( & -6 A_{0 x} s_{1}^{3} s_{2}-3 A_{1 x} s_{1}^{4}-6 A_{2 t} r s_{1}^{3} s_{2}-12 A_{2 t} s_{1}^{4} \\
& +12 A_{2 x} r^{2} s_{1}^{3} s_{2}+9 A_{2 x} r s_{1}^{4}+36 r_{x}^{2} r s_{1}^{2} s_{2}^{2}
\end{aligned}
$$




$$
\begin{aligned}
& -36 r_{x}^{2} s_{1}^{3} s_{2}-36 r_{x} s_{1 x} r s_{1}^{2} s_{2}+36 r_{x} s_{2 x} r s_{1}^{3} \\
& -6 r_{x} A_{1} s_{1}^{3} s_{2}+18 r_{x} A_{2} r s_{1}^{3} s_{2}-3 r_{x} A_{2} s_{1}^{4} \\
& -12 r_{x} r s_{1} s_{2} s_{3}+12 s_{1 t} s_{1} s_{3}-4 A_{1} A_{2} r s_{1}^{3} s_{2} \\
& -5 A_{1} A_{2} s_{1}^{4}+8 A_{2}^{2} r^{2} s_{1}^{3} s_{2}+7 A_{2}^{2} r s_{1}^{4}-12 B_{3} r s_{1}^{3} s_{2} \\
& -15 B_{3} s_{1}^{4}+48 B_{4} r^{2} s_{1}^{3} s_{2}+51 B_{4} r s_{1}^{4} \\
& -120 B_{5} r^{3} s_{1}^{3} s_{2}-105 B_{5} r^{2} s_{1}^{4}-2 r s_{1}^{3} s_{2}^{2} \\
& \left.+r s_{3}^{2}+5 s_{1}^{4} s_{2}\right) /\left(6 s_{1}^{2}\right) .
\end{aligned}
$$

Equation (15) provides the conditions

$$
\begin{aligned}
A_{0 t}= & 6 A_{0 x} r+6 A_{2 t} r^{2}-6 A_{2 x} r^{3}-7 A_{0} A_{1} \\
& +9 A_{0} A_{2} r+5 A_{1}^{2} r-8 A_{1} A_{2} r^{2}+A_{2}^{2} r^{3}-36 B_{1} \\
& \left.+54 B_{2} r-54 B_{3} r^{2}+36 B_{4} r^{3}-r s_{1}\right) / 6 \\
B_{0}=( & -A_{0}^{2}+2 A_{0} A_{1} r-2 A_{0} A_{2} r^{2}-A_{1}^{2} r^{2} \\
& +2 A_{1} A_{2} r^{3}-A_{2}^{2} r^{4}+12 B_{1} r-12 B_{2} r^{2}+12 B_{3} r^{3} \\
& \left.-12 B_{4} r^{4}+12 B_{5} r^{5}\right) / 12 .
\end{aligned}
$$

Comparing the mixed derivatives $\left(G_{1 t t}\right)_{x}=\left(G_{1 t x}\right)_{t},\left(G_{1 x x}\right)_{t}=$ $\left(G_{1 t x}\right)_{x}$, and $\left(F_{x x x}\right)_{t}=\left(F_{t}\right)_{x x x}$, one gets the conditions

$$
\begin{aligned}
A_{1 x x}=( & -33 A_{1 x} A_{2} s_{1}^{2}-18 A_{2 t x} s_{1}^{2}-108 A_{2 t} r_{x} s_{1} s_{2} \\
& +24 A_{2 t} A_{2} s_{1}^{2}+18 A_{2 t} s_{3}+54 A_{2 x x} r s_{1}^{2} \\
& +108 A_{2 x} r_{x} r s_{1} s_{2}+18 A_{2 x} r_{x} s_{1}^{2}-30 A_{2 x} A_{1} s_{1}^{2} \\
& +102 A_{2 x} A_{2} r s_{1}^{2}-18 A_{2 x} r s_{3}-90 B_{3 x} s_{1}^{2} \\
& +54 B_{4 t} s_{1}^{2}+306 B_{4 x} r s_{1}^{2}-270 B_{5 t} r s_{1}^{2}-630 B_{5 x} r^{2} s_{1}^{2} \\
& -36 r_{x} A_{1} A_{2} s_{1} s_{2}+72 r_{x} A_{2}^{2} r s_{1} s_{2}+27 r_{x} A_{2}^{2} s_{1}^{2} \\
& -108 r_{x} B_{3} s_{1} s_{2}+432 r_{x} B_{4} r s_{1} s_{2}+252 r_{x} B_{4} s_{1}^{2} \\
& -1080 r_{x} B_{5} r^{2} s_{1} s_{2}-1260 r_{x} B_{5} r s_{1}^{2}+36 r_{x} s_{1} s_{2}^{2} \\
& -18 s_{1 x} s_{1} s_{2}+30 s_{2 x} s_{1}^{2}+45 A_{0} B_{5} s_{1}^{2}-5 A_{1} A_{2}^{2} s_{1}^{2} \\
& +6 A_{1} A_{2} s_{3}-45 A_{1} B_{5} r s_{1}^{2}+10 A_{2}^{3} r s_{1}^{2}-12 A_{2}^{2} r s_{3} \\
& -15 A_{2} B_{3} s_{1}^{2}+60 A_{2} B_{4} r s_{1}^{2}-105 A_{2} B_{5} r^{2} s_{1}^{2} \\
& +5 A_{2} s_{1}^{2} s_{2}+18 B_{3} s_{3}-72 B_{4} r s_{3}+180 B_{5} r^{2} s_{3} \\
& \left.-6 s_{2} s_{3}\right) /\left(18 s_{1}^{2}\right),
\end{aligned}
$$

$$
\begin{aligned}
A_{2 t t}=( & 36 A_{2 t x} r s_{1}+72 A_{2 t} r_{x} s_{1}-18 A_{2 x x} r^{2} s_{1} \\
& -72 A_{2 x} r_{x} r s_{1}-3 A_{2 x} A_{0} s_{1}+3 A_{2 x} A_{1} r s_{1} \\
& -3 A_{2 x} A_{2} r^{2} s_{1}-18 B_{3 t} s_{1}+18 B_{3 x} r s_{1} \\
& +72 B_{4 t} r s_{1}-72 B_{4 x} r^{2} s_{1}-180 B_{5 t} r^{2} s_{1} \\
& +180 B_{5 x} r^{3} s_{1}+24 r_{x} A_{1} A_{2} s_{1}-48 r_{x} A_{2}^{2} r s_{1} \\
& +72 r_{x} B_{3} s_{1}-288 r_{x} B_{4} r s_{1}+720 r_{x} B_{5} r^{2} s_{1}-6 r_{x} s_{1} s_{2} \\
& -3 s_{1 x} s_{1}+3 A_{0} A_{2}^{2} s_{1}-12 A_{0} B_{4} s_{1}+60 A_{0} B_{5} r s_{1} \\
& +4 A_{1}^{2} A_{2} s_{1}-19 A_{1} A_{2}^{2} r s_{1}+6 A_{1} B_{3} s_{1}-12 A_{1} B_{4} r s_{1} \\
& +19 A_{2}^{3} r^{2} s_{1}+18 A_{2} B_{2} s_{1} \\
& -66 A_{2} B_{3} r s_{1}+144 A_{2} B_{4} r^{2} s_{1} \\
& \left.-240 A_{2} B_{5} r^{3} s_{1}+2 A_{2} s_{1}^{2}+s_{3}\right) /\left(18 s_{1}\right),
\end{aligned}
$$$$
A_{2 t x}=\left(-6 A_{1 x} A_{2} s_{1}^{2} s_{2}-72 A_{2 t} r_{x} s_{1} s_{2}^{2}+90 A_{2 t} s_{1 x} s_{1} s_{2}\right.
$$$$
-90 A_{2 t} s_{2 x} s_{1}^{2}-24 A_{2 t} A_{2} s_{1}^{2} s_{2}+12 A_{2 t} s_{2} s_{3}
$$$$
+18 A_{2 x x} r s_{1}^{2} s_{2}-9 A_{2 x x} s_{1}^{3}+72 A_{2 x} r_{x} r s_{1} s_{2}^{2}
$$$$
+18 A_{2 x} r_{x} s_{1}^{2} s_{2}-90 A_{2 x} s_{1 x} r s_{1} s_{2}+90 A_{2 x} s_{2 x} r s_{1}^{2}
$$$$
-6 A_{2 x} A_{1} s_{1}^{2} s_{2}+48 A_{2 x} A_{2} r s_{1}^{2} s_{2}-18 A_{2 x} A_{2} s_{1}^{3}
$$$$
-12 A_{2 x} r s_{2} s_{3}-18 B_{3 x} s_{1}^{2} s_{2}+72 B_{4 x} r s_{1}^{2} s_{2}
$$$$
-27 B_{4 x} s_{1}^{3}+54 B_{5 t} s_{1}^{3}-180 B_{5 x} r^{2} s_{1}^{2} s_{2}
$$$$
+81 B_{5 x} r s_{1}^{3}-24 r_{x} A_{1} A_{2} s_{1} s_{2}^{2}+48 r_{x} A_{2}^{2} r s_{1} s_{2}^{2}
$$$$
+12 r_{x} A_{2}^{2} s_{1}^{2} s_{2}-72 r_{x} B_{3} s_{1} s_{2}^{2}+288 r_{x} B_{4} r s_{1} s_{2}^{2}
$$$$
+72 r_{x} B_{4} s_{1}^{2} s_{2}-720 r_{x} B_{5} r^{2} s_{1} s_{2}^{2}-360 r_{x} B_{5} r s_{1}^{2} s_{2}
$$$$
+135 r_{x} B_{5} s_{1}^{3}+30 s_{1 x} A_{1} A_{2} s_{1} s_{2}-60 s_{1 x} A_{2}^{2} r s_{1} s_{2}
$$$$
+90 s_{1 x} B_{3} s_{1} s_{2}-360 s_{1 x} B_{4} r s_{1} s_{2}
$$$$
+900 s_{1 x} B_{5} r^{2} s_{1} s_{2}-30 s_{2 x} A_{1} A_{2} s_{1}^{2}+60 s_{2 x} A_{2}^{2} r s_{1}^{2}
$$$$
-90 s_{2 x} B_{3} s_{1}^{2}+360 s_{2 x} B_{4} r s_{1}^{2}-900 s_{2 x} B_{5} r^{2} s_{1}^{2}
$$$$
-8 A_{1} A_{2}^{2} s_{1}^{2} s_{2}+4 A_{1} A_{2} s_{2} s_{3}+18 A_{1} B_{5} s_{1}^{3}
$$$$
+16 A_{2}^{3} r s_{1}^{2} s_{2}-4 A_{2}^{3} s_{1}^{3}-8 A_{2}^{2} r s_{2} s_{3}
$$$$
-24 A_{2} B_{3} s_{1}^{2} s_{2}+96 A_{2} B_{4} r s_{1}^{2} s_{2}-18 A_{2} B_{4} s_{1}^{3}
$$$$
-240 A_{2} B_{5} r^{2} s_{1}^{2} s_{2}+54 A_{2} B_{5} r s_{1}^{3}+12 B_{3} s_{2} s_{3}
$$$$
\left.-48 B_{4} r s_{2} s_{3}+120 B_{5} r^{2} s_{2} s_{3}\right) /\left(18 s_{1}^{2} s_{2}\right) \text {. }
$$ 
3.2.2. Case $s_{1}=0$. From (35), one finds the condition

$$
s_{2}=0 \text {. }
$$

Equation (15) gives the conditions

$$
\begin{aligned}
A_{0 t}=( & 6 A_{0 x} r+6 A_{2 t} r^{2}-6 A_{2 x} r^{3}-7 A_{0} A_{1} \\
& +9 A_{0} A_{2} r+5 A_{1}^{2} r-8 A_{1} A_{2} r^{2}+A_{2}^{2} r^{3} \\
& \left.-36 B_{1}+54 B_{2} r-54 B_{3} r^{2}+36 B_{4} r^{3}\right) / 6 \\
B_{0}=( & A_{0}^{2}+2 A_{0} A_{1} r-2 A_{0} A_{2} r^{2}-A_{1}^{2} r^{2} \\
& +2 A_{1} A_{2} r^{3}-A_{2}^{2} r^{4}+12 B_{1} r-12 B_{2} r^{2} \\
& \left.+12 B_{3} r^{3}-12 B_{4} r^{4}+12 B_{5} r^{5}\right) / 12 .
\end{aligned}
$$

From the mixed derivative $\left(G_{1 x x}\right)_{t}=\left(G_{1 t x}\right)_{x}$, one finds the condition

$$
\begin{aligned}
A_{2 t t}=( & 36 A_{2 t x} r+72 A_{2 t} r_{x}-18 A_{2 x x} r^{2} \\
& -72 A_{2 x} r_{x} r-3 A_{2 x} A_{0}+3 A_{2 x} A_{1} r \\
& -3 A_{2 x} A_{2} r^{2}-18 B_{3 t}+18 B_{3 x} r+72 B_{4 t} r \\
& -72 B_{4 x} r^{2}-180 B_{5 t} r^{2}+180 B_{5 x} r^{3} \\
& +24 r_{x} A_{1} A_{2}-48 r_{x} A_{2}^{2} r+72 r_{x} B_{3}-288 r_{x} B_{4} r \\
& +720 r_{x} B_{5} r^{2}+3 A_{0} A_{2}^{2}-12 A_{0} B_{4}+60 A_{0} B_{5} r \\
& +4 A_{1}^{2} A_{2}-19 A_{1} A_{2}^{2} r+6 A_{1} B_{3}-12 A_{1} B_{4} r \\
& +19 A_{2}^{3} r^{2}+18 A_{2} B_{2}-66 A_{2} B_{3} r \\
& \left.+144 A_{2} B_{4} r^{2}-240 A_{2} B_{5} r^{3}\right) / 18
\end{aligned}
$$

The relation $\left(G_{1 t t}\right)_{x}=\left(G_{1 t x}\right)_{t}$ becomes

$$
18\left(F_{x} G_{1 t}-F_{x} G_{1 x} r-F_{x} r_{x} G_{1}-G_{1 x} K\right) s_{4}+G_{1} K s_{5}=0,
$$

where

$$
\begin{aligned}
s_{4}= & 3 A_{2 t}-3 A_{2 x} r+A_{1} A_{2}-2 A_{2}^{2} r+3 B_{3} \\
& -12 B_{4} r+30 B_{5} r^{2}, \\
s_{5}= & 18 A_{1 x x}+27 A_{1 x} A_{2}-36 A_{2 x x} r+24 A_{2 x} A_{1} \\
& -102 A_{2 x} A_{2} r+72 B_{3 x}-54 B_{4 t}-234 B_{4 x} r \\
& +270 B_{5 t} r+450 B_{5 x} r^{2}-15 r_{x} A_{2}^{2}-180 r_{x} B_{4} \\
& +900 r_{x} B_{5} r+6 s_{4 x}-45 A_{0} B_{5}+13 A_{1} A_{2}^{2} \\
& +45 A_{1} B_{5} r-26 A_{2}^{3} r+39 A_{2} B_{3}-156 A_{2} B_{4} r \\
& +345 A_{2} B_{5} r^{2}-8 A_{2} s_{4} .
\end{aligned}
$$

The relation $\left(A_{2 t}\right)_{t}-A_{2 t t}=0$ provides the condition

$$
s_{4 t}=\frac{\left(12 r_{x} s_{4}+3 s_{4 x} r+A_{1} s_{4}-2 A_{2} r s_{4}\right)}{3} .
$$

Further study depends on $s_{4}$.

(i) Case $s_{4} \neq 0$

From (46), one gets the derivative

$$
g_{1 t}=\frac{\left(18\left(F_{x} G_{1 x} r+F_{x} r_{x} G_{1}+G_{1 x} K\right) s_{4}-G_{1} K s_{5}\right)}{\left(18 F_{x} s_{4}\right)} .
$$

Differentiating $g_{1 t}$ with respect to $x$, one obtains the derivative

$$
F_{x}=\frac{\left(K s_{6}\right)}{\left(108 s_{4}^{3}\right)}
$$

where

$$
\begin{aligned}
s_{6}= & 324 A_{2 x} s_{4}^{2}-36 s_{4 x} s_{5}+36 s_{5 x} s_{4}+108 A_{2}^{2} s_{4}^{2} \\
& +324 B_{4} s_{4}^{2}-1620 B_{5} r s_{4}^{2}-s_{5}^{2} .
\end{aligned}
$$

The relations $\left(F_{x}\right)_{t}=\left(F_{t}\right)_{x},\left(G_{1 t}\right)_{t}=G_{1 t t},\left(F_{x x x}\right)_{t}=$ $\left(F_{t}\right)_{x x x}$, and $\left(F_{x}\right)_{x x}=F_{x x x}$ provide the conditions

$$
\begin{aligned}
s_{6 t}= & \left(30 r_{x} s_{6}+3 s_{6 x} r+2 A_{1} s_{6}-4 A_{2} r s_{6}+108 A_{2} s_{4}^{3}\right. \\
& \left.+18 s_{4}^{2} s_{5}\right) / 3 \\
s_{5 t}= & -108 A_{1 x} s_{4}^{2}-108 A_{2 x} r s_{4}^{2}+108 r_{x} A_{2} s_{4}^{2} \\
& +180 r_{x} s_{4} s_{5}+36 s_{4 x} r s_{5}-36 A_{1} A_{2} s_{4}^{2} \\
& +12 A_{1} s_{4} s_{5}-36 A_{2}^{2} r s_{4}^{2}-24 A_{2} r s_{4} s_{5} \\
& -108 B_{3} s_{4}^{2}+108 B_{4} r s_{4}^{2}+540 B_{5} r^{2} s_{4}^{2}+r s_{5}^{2} \\
& \left.+r s_{6}-144 s_{4}^{3}\right) /\left(36 s_{4}\right),
\end{aligned}
$$$$
A_{2 x x}=\left(-5832 A_{2 x} A_{2} s_{4}^{3}-8748 B_{4 x} s_{4}^{3}+17496 B_{5 t} s_{4}^{3}\right.
$$$$
+26244 B_{5 x} r s_{4}^{3}+43740 r_{x} B_{5} s_{4}^{3}-126 s_{4 x} s_{6}
$$$$
+45 s_{6 x} s_{4}+5832 A_{1} B_{5} s_{4}^{3}-1296 A_{2}^{3} s_{4}^{3}
$$$$
-5832 A_{2} B_{4} s_{4}^{3}+17496 A_{2} B_{5} r s_{4}^{3}
$$$$
\left.+12 A_{2} s_{4} s_{6}-s_{5} s_{6}\right) /\left(2916 s_{4}^{3}\right),
$$$$
s_{6 x x}=\left(-324 A_{2 x} s_{4}^{2} s_{6}+2916 s_{4 x x} s_{4} s_{6}-11664 s_{4 x}^{2} s_{6}\right.
$$$$
+5832 s_{4 x} s_{6 x} s_{4}+1944 s_{4 x} A_{2} s_{4} s_{6}-162 s_{4 x} s_{5} s_{6}
$$$$
-648 s_{6 x} A_{2} s_{4}^{2}+54 s_{6 x} s_{4} s_{5}-108 A_{2}^{2} s_{4}^{2} s_{6}
$$$$
\left.+18 A_{2} s_{4} s_{5} s_{6}-104976 B_{5} s_{4}^{5}+s_{6}^{2}\right) /\left(972 s_{4}^{2}\right) .
$$ 
(ii) Case $s_{4}=0$

From (46), one gets the condition

$$
s_{5}=0
$$

Comparing the mixed derivative $\left(F_{x x x}\right)_{t}=\left(F_{t}\right)_{x x x}$, one arrives at the condition

$$
\begin{aligned}
A_{2 x x}=( & -18 A_{2 x} A_{2}-27 B_{4 x}+54 B_{5 t} \\
& +81 B_{5 x} r+135 r_{x} B_{5}+18 A_{1} B_{5}-4 A_{2}^{3} \\
& \left.-18 A_{2} B_{4}+54 A_{2} B_{5} r\right) / 9 .
\end{aligned}
$$

All obtained results can be summarized in the following theorems.

Theorem 2. Sufficient conditions for (8) to be linearizable via the generalized linearizing transformation (5) with $F_{x}=0$ are equations (18), (22), (24), (25), (28), (29), (30), and (31).

Corollary 3. Provided that the sufficient conditions in Theorem 2 are satisfied, the transformation (5) mapping equation (8) to a linear equation (6) is obtained by solving the compatible system of equations (21), (23), and (26) for the functions $F(t), G_{1}(t, x)$, and $G_{2}(t, x)$.

Theorem 4. Sufficient conditions for equation (8) to be linearizable via the generalized linearizing transformation (5) with $F_{x} \neq 0$ are as follows.

(a) If $s_{1} \neq 0$, then the conditions are (18), (40), (41), and (42).

(b) If $s_{1}=0, s_{4} \neq 0$, then the conditions are (18), (43), (44), (48), and (52).

(c) If $s_{1}=0, s_{4}=0$, then the conditions are (18), (43), (44), (53), and (54).

Corollary 5. Provided that the sufficient conditions in Theorem 4 are satisfied, the transformation (5) mapping equation (8) to a linear equation (6) is obtained by solving the following compatible system of equations for the functions $F(t, x), G_{1}(t, x)$, and $G_{2}(t, x)$ :

(a) (16), (17), (34), (37), and (38);

(b) (16), (17), (34), (49), and (50);

(c) (16), (17), (19), (32), (33), and (34).

\section{Examples}

For understanding the procedure of using the linearization theorems, we consider the following examples.
Example 1. Consider the nonlinear third-order ordinary differential equation

$$
\begin{aligned}
3 x^{\prime 4} t^{2} & +2 x^{\prime 3} t(3 t+2 x)+3 x^{\prime 2} x^{\prime \prime} t^{2} x \\
& +x^{\prime 2}\left(3 t^{2}+8 t x+3 x^{2}\right)+2 x^{\prime} x^{\prime \prime} t x(t+2 x) \\
& -x^{\prime} x^{\prime \prime \prime} t^{2} x^{2}+2 x^{\prime} x(2 t+3 x)+3 x^{\prime \prime 2} t^{2} x^{2} \\
& +x^{\prime \prime} t x(-t+4 x)-x^{\prime \prime \prime} t^{2} x^{2}+3 x^{2}=0 .
\end{aligned}
$$

It is an equation of the form (8) in Theorem 1 with the coefficients

$$
\begin{gathered}
A_{2}=-\frac{3}{x}, \quad A_{1}=-\frac{2(t+2 x)}{t x}, \\
A_{0}=\frac{t-4 x}{t x}, \quad B_{5}=0, \quad B_{4}=-\frac{3}{x^{2}}, \\
B_{3}=-\frac{2(3 t+2 x)}{t x^{2}}, \quad B_{2}=-\frac{3 t^{2}+8 t x+3 x^{2}}{t^{2} x^{2}}, \\
B_{1}=-\frac{2(2 t+3 x)}{t^{2} x}, \quad B_{0}=-\frac{3}{t^{2}} \\
r=1, \quad h_{1}=-\frac{12}{t}, \quad h_{2}=-\frac{450}{t^{2}} .
\end{gathered}
$$

One can check that these coefficients obey the conditions in Theorem 2. Thus, (55) is linearizable via a generalized linearizing transformation. For finding the functions $F, G_{1}$, and $G_{2}$, we have to solve equations in Corollary 3, which become

$$
\begin{gathered}
F_{x}=0, \quad F_{t}=K, \\
G_{1 x}=\frac{G_{1}}{x}, \quad G_{1 t t}=\frac{\left(5 G_{1 t}^{2} t^{2}-4 G_{1 t} G_{1} t-G_{1}^{2}\right)}{\left(3 G_{1} t^{2}\right)}, \\
K_{x}=0, \quad K_{t}=\frac{\left(4 K G_{1 t} t-G_{1}\right)}{\left(3 G_{1} t\right)} .
\end{gathered}
$$

From the first equation of system (58), we get $G_{1}=x f(t)$, and choosing $f(t)=t$, we have

$$
G_{1}=x t
$$

and this solution satisfies the second equation. Since $r=1$, then we obtain

$$
G_{2}=x t
$$

System (59) becomes

$$
K_{x}=0, \quad K_{t}=0,
$$

and one can take the simplest solution

$$
K=1 .
$$

System (90) becomes

$$
F_{x}=0, \quad F_{t}=1,
$$


so that we get the particular solution

$$
F=t
$$

Thus, one obtains the linearizing transformation

$$
X=t, \quad d T=t x\left(x^{\prime}+1\right) d t .
$$

Hence, (55) is mapped by the transformation of (66) into the linear equation (6).

Example 2. Consider the nonlinear third-order ordinary differential equation

$$
\begin{aligned}
3 x^{15} t^{2} & +x^{\prime 4} t(3 t+4 x)+x^{\prime 3} x(4 t+3 x) \\
& +x^{\prime 2} x^{\prime \prime} t x(3 t+x)+3 x^{\prime 2} x^{2}+4 x^{\prime} x^{\prime \prime} t x^{2} \\
& -x^{\prime} x^{\prime \prime \prime} t^{2} x^{2}+3 x^{\prime \prime 2} t^{2} x^{2}=0
\end{aligned}
$$

It is an equation of the form (8) in Theorem 1 with the coefficients

$$
\begin{gathered}
A_{2}=-\frac{(3 t+x)}{t x}, \quad A_{1}=-\frac{4}{t}, \quad A_{0}=0, \\
B_{5}=-\frac{3}{x^{2}}, \quad B_{4}=-\frac{(3 t+4 x)}{t x^{2}}, \\
B_{3}=-\frac{(4 t+3 x)}{t^{2} x}, \quad B_{2}=-\frac{3}{t^{2}}, \\
B_{1}=0, \quad B_{0}=0, \quad r=0, \\
s_{1}=-\frac{2}{t^{2}}, \quad s_{2}=\frac{1}{t^{2}}, \quad s_{3}=\frac{12(t-x)}{t^{5} x} .
\end{gathered}
$$

One can check that these coefficients obey the conditions in Theorem 4(a). Thus, (67) is linearizable via a generalized linearizing transformation. For finding the functions $F, G_{1}$, and $G_{2}$, we have to solve equations in Corollary 5(a), which become

$$
\begin{gathered}
F_{x}=K, \quad F_{t}=K, \\
G_{1 t}=\frac{\left(G_{1 x} t x-G_{1} t+G_{1} x\right)}{(t x)}, \\
G_{1 x x}=\left(G_{1 x}^{2} t x^{2}+4 G_{1 x} G_{1} t x-4 G_{1 x} G_{1} x^{2}\right. \\
\left.-5 G_{1}^{2} t+4 G_{1}^{2} x\right) /\left(3 G_{1} t x^{2}\right), \\
K_{x}=\frac{\left(4 K\left(G_{1 x} x-G_{1}\right)\right)}{\left(3 G_{1} x\right)} \\
K_{t}=\frac{\left(4 K\left(G_{1 x} x-G_{1}\right)\right)}{\left(3 G_{1} x\right)} .
\end{gathered}
$$

From the first equation of system (70), one can take the particular solution

$$
G_{1}=t x
$$

and this solution satisfies the second equation. Since $r=0$, then we obtain

$$
G_{2}=0 \text {. }
$$

System (71) becomes

$$
K_{x}=0, \quad K_{t}=0,
$$

and one can take the simplest solution

$$
K=1
$$

System (69) becomes

$$
F_{x}=1, \quad F_{t}=1,
$$

so that we get the particular solution

$$
F=t+x
$$

Thus, one obtains the linearizing transformation

$$
X=t+x, \quad d T=t x x^{\prime} d t .
$$

Hence, (67) is mapped by the transformation of (78) into the linear equation (6).

Example 3. Consider the nonlinear third-order ordinary differential equation

$$
3 x^{\prime \prime 2} x^{2}-3 x^{\prime 4}-3 x^{\prime 2} x^{\prime \prime} x-x^{\prime} x^{\prime \prime \prime} x^{2}=0 .
$$

Note that this equation can be reduced to an autonomous equation by the substitution

$$
x=t v(s), \quad s=\ln (t),
$$

and then to the second-order ordinary differential equation

$$
\begin{aligned}
y^{\prime \prime} z^{2} & y^{2}(z+y) \\
= & y^{\prime 2} z^{2} y(-2 z+y)-3 y^{\prime} z y\left(z^{2}+y^{2}\right) \\
& \quad-3 z^{4}-14 z^{3} y-20 z^{2} y^{2}-15 z y^{3}-3 y^{4},
\end{aligned}
$$

where $y=y(z)$. However, the latter equation is not linearizable by point transformations.

Equation (79) is an equation of the form (8) in Theorem 1 with the coefficients

$$
\begin{aligned}
& A_{2}=\frac{3}{x}, \quad A_{1}=0, \quad A_{0}=0, \\
& B_{5}=0, \quad B_{4}=\frac{3}{x^{2}}, \quad B_{3}=0, \quad B_{2}=0, \\
& B_{1}=0, \quad B_{0}=0, \quad r=0, \\
& s_{1}=0, \quad s_{2}=0, \quad s_{4}=0, \quad s_{5}=0 .
\end{aligned}
$$

One can check that these coefficients obey the conditions in Theorem 4(c). Thus, (79) is linearizable via a generalized 
linearizing transformation. For finding the functions $F, G_{1}$, and $G_{2}$, we have to solve equations in Corollary $5(c)$, which become

$$
\begin{gathered}
F_{t}=K, \\
F_{x x x}=\left(6 F_{x x} F_{x} G_{1 t} G_{1} K x^{2}+18 F_{x x} G_{1 x} G_{1} K^{2} x^{2}\right. \\
-F_{x}^{3} G_{1 t}^{2} x^{2}-6 F_{x}^{2} G_{1 t} G_{1 x} K x^{2}-9 F_{x} G_{1 x}^{2} K^{2} x^{2} \\
\left.-9 F_{x} G_{1}^{2} K^{2}\right) /\left(6 G_{1}^{2} K^{2} x^{2}\right), \\
G_{1 t t}=\frac{\left(5 G_{1 t}^{2}\right)}{\left(3 G_{1}\right)}, \\
G_{1 t x}=\frac{\left(G_{1 t}\left(-F_{x} G_{1 t} x+6 G_{1 x} K x+3 G_{1} K\right)\right)}{\left(3 G_{1} K x\right)} \\
G_{1 x x}=\left(6 F_{x x} G_{1 t} G_{1} K x^{2}-F_{x}^{2} G_{1 t}^{2} x^{2}-6 F_{x} G_{1 t} G_{1 x} K x^{2}\right. \\
\left.+9 G_{1 x}^{2} K^{2} x^{2}-9 G_{1}^{2} K^{2}\right) /\left(6 G_{1} K^{2} x^{2}\right), \\
K_{x}=\frac{\left(F_{x} G_{1 t} x+3 G_{1 x} K x+3 G_{1} K\right)}{\left(3 G_{1} x\right)}, \\
K_{t}=\frac{\left(4 G_{1 t} K\right)}{\left(3 G_{1}\right)} .
\end{gathered}
$$

From the first equation of system (84), one can take the particular solution

$$
G_{1}=x
$$

and this solution satisfies the second and third equations. Since $r=0$, then we obtain

$$
G_{2}=0 .
$$

System (85) becomes

$$
K_{x}=\frac{(2 K)}{x}, \quad K_{t}=0,
$$

and one can take the particular solution

$$
K=x^{2} .
$$

System (83) becomes

$$
F_{x}=x^{2}, \quad F_{x x x}=\frac{\left(3\left(F_{x x} x-F_{x}\right)\right)}{x^{2}},
$$

so that one obtains the particular solution of the first equation as

$$
F=t x^{2}
$$

and this solution satisfies the second equation. Then we get the linearizing transformation

$$
X=t x^{2}, \quad d T=x x^{\prime} d t .
$$

Hence, equation (79) is mapped by the transformation of (92) into the linear equation (6).

\section{Conclusion}

This paper is devoted to find the conditions which allow the third-order ordinary differential equation to be transformed into the simplest linear equation. Necessary conditions which guarantee that the third-order ordinary differential equation can be linearized are found in Theorem 1 . Theorems 2 and 4 are sufficient conditions for the linearization problem. The linearizing transformation can be found by solving the compatible system in Corollaries 3 and 5. Finally, some examples are provided to demonstrate our procedure.

\section{Conflict of Interests}

The authors declare that there is no conflict of interests regarding the publication of this paper.

\section{Acknowledgment}

This research was financially supported by the National Research Council of Thailand under Grant no. R2557B057.

\section{References}

[1] S. V. Meleshko, Methods for Constructing Exact Solutions of Partial Differential Equations, Mathematical and Analytical Techniques with Applications to Engineering, Springer, New York, NY, USA, 2005.

[2] N. H. Ibragimov, Elementary Lie Group Analysis and Ordinary Differential Equations, John Wiley \& Sons, Chichester, UK, 1999.

[3] L. G. S. Duarte, I. C. Moreira, and F. C. Santos, "Linearization under nonpoint transformations," Journal of Physics A: Mathematical and General, vol. 27, no. 19, pp. L739-L743, 1994.

[4] C. Muriel and J. L. Romero, "Nonlocal transformations and linearization of second-order ordinary differential equations," Journal of Physics A: Mathematical and Theoretical, vol. 43, no. 43, Article ID 434025, 13 pages, 2010.

[5] M. T. Mustafa, A. Y. Al-Dweik, and R. Mara'beh, "On the linearization of second-order ordinary differential equations to the Laguerre form via generalized Sundman transformations," SIGMA, vol. 9, article 41, 10 pages, 2013.

[6] W. Nakpim and S. V. Meleshko, "Linearization of third-order ordinary differential equations by generalized Sundman transformations: the case $X^{\prime \prime \prime}+\alpha X=0$," Communications in Nonlinear Science and Numerical Simulation, vol. 15, no. 7, pp. 1717-1723, 2010.

[7] L. M. Berkovich, "The method of an exact linearization of $n$-order ordinary differential equations," Journal of Symbolic Computation, vol. 27, no. 5, pp. 501-519, 1999.

[8] N. Euler, T. Wolf, P. G. L. Leach, and M. Euler, "Linearisable third-order ordinary differential equations and generalised Sundman transformations: the case $X^{\prime \prime \prime}=0$," Acta Applicandae Mathematicae. An International Survey Journal on Applying Mathematics and Mathematical Applications, vol. 76, no. 1, pp. 89-115, 2003.

[9] L. M. Berkovich, "The integration of ordinary differential equations: factorization and transformations," Mathematics and Computers in Simulation, vol. 57, no. 3-5, pp. 175-195, 2001.

[10] L. Berkovich, Factorization and Transformations of Differential Equations, Methods and Applications, R \& C Dynamics, Moscow, Russia, 2002. 
[11] S. Suksern and S. Tammakun, "Linearization of nonlinear fourth-order ordinary differential equations by a generalized sundman transformation," Far East Journal of Applied Mathematics, vol. 86, no. 3, pp. 183-210, 2014.

[12] V. K. Chandrasekar and M. Lakshmanan, "A unification in the theory of linearization of second-order nonlinear ordinary differential equations," Journal of Physics A: Mathematical and General, vol. 39, no. 3, pp. L69-L76, 2006.

[13] V. K. Chandrasekar, M. Senthilvelan, and M. Lakshmanan, "On the complete integrability and linearization of certain secondorder nonlinear ordinary differential equations," Proceedings of The Royal Society of London A Mathematical, Physical and Engineering Sciences, vol. 461, no. 2060, pp. 2451-2476, 2005.

[14] V. K. Chandrasekar, M. Senthilvelan, and M. Lakshmanan, "On the complete integrability and linearization of nonlinear ordinary differential equations. II. Third-order equations," Proceedings of the Royal Society A, vol. 462, no. 2070, pp. 1831-1852, 2006. 


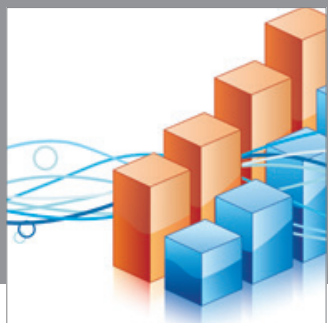

Advances in

Operations Research

mansans

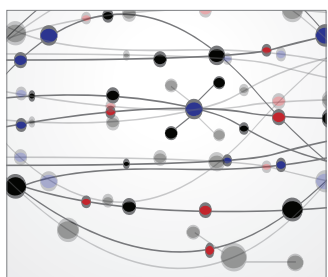

The Scientific World Journal
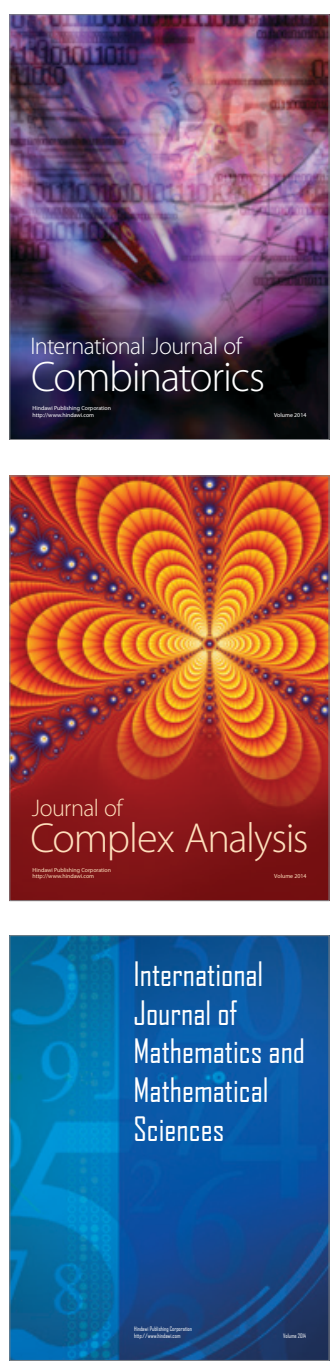
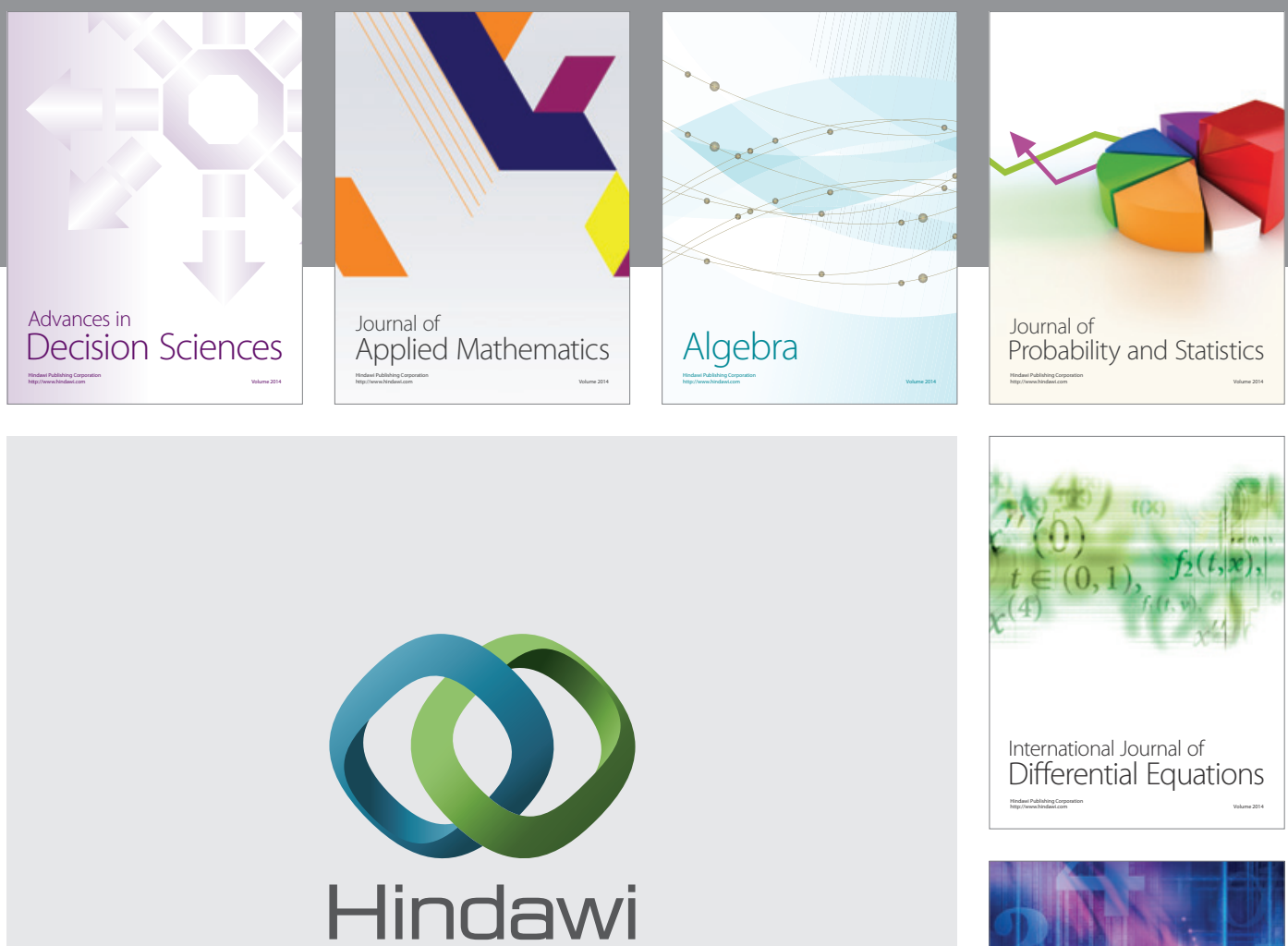

Submit your manuscripts at http://www.hindawi.com
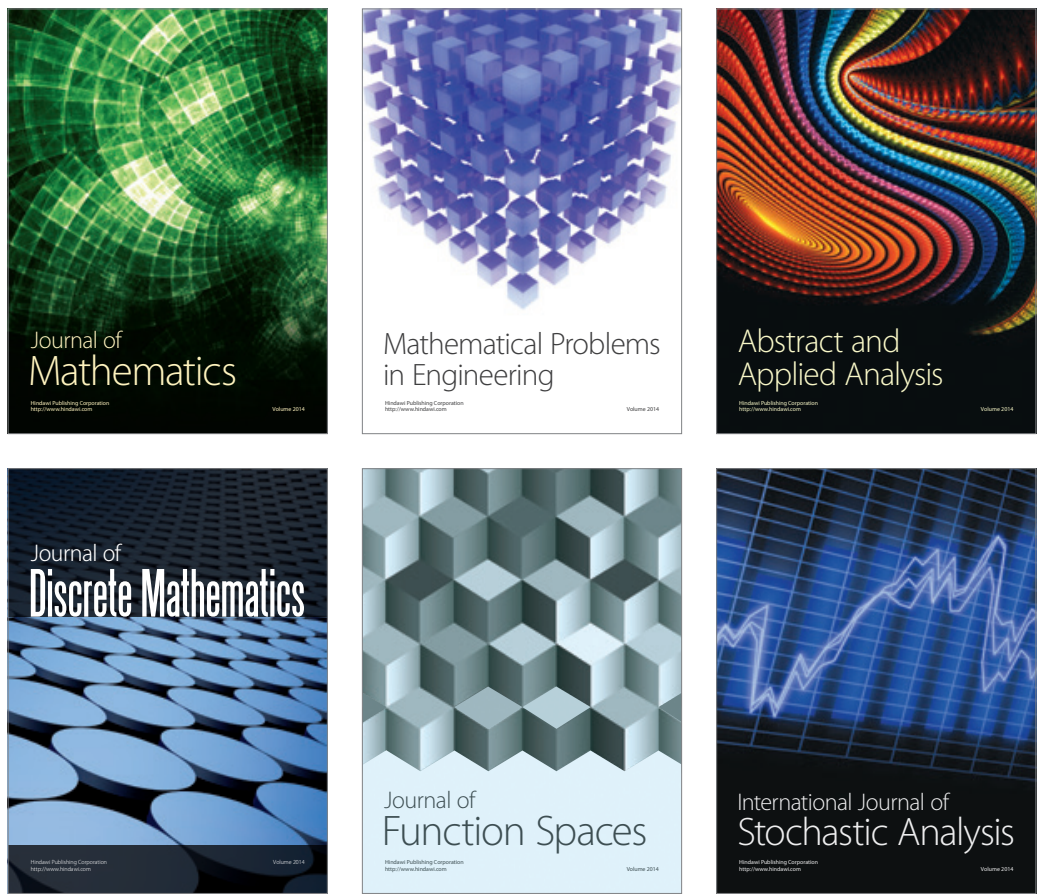

Journal of

Function Spaces

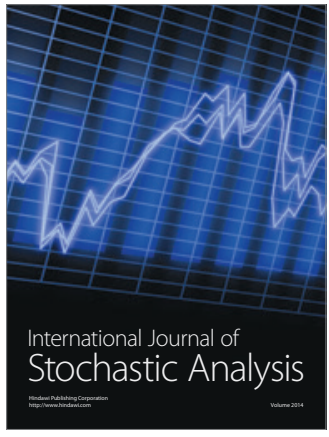

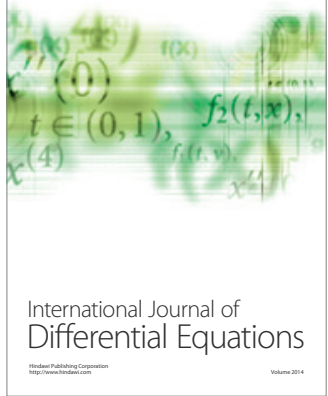
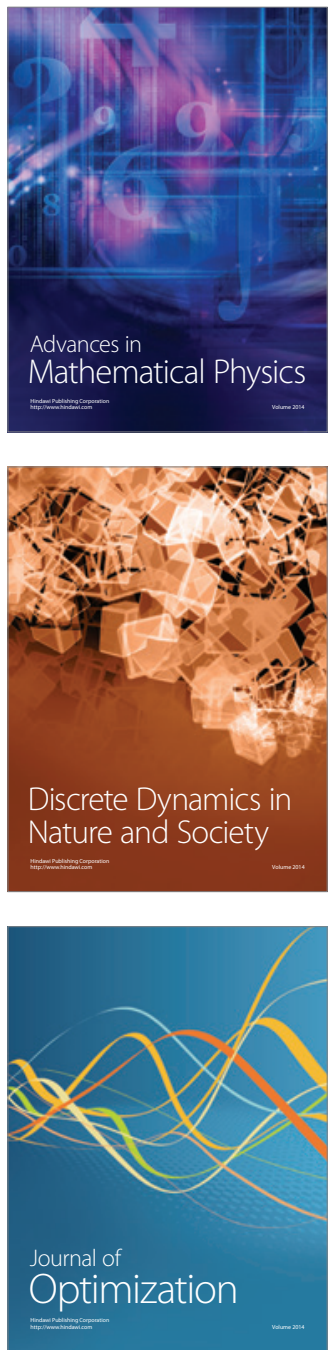